\title{
Quantum oscillations of resistivity in bismuth nanowires
}

\author{
E. Condrea ${ }^{1,2}$ and A. Gilewski ${ }^{2}$ \\ ${ }^{1}$ Institute of Electronic Engineering and Industrial Technologies, Academy of Sciences of Moldova \\ 3/3 Academiei Str. MD 2028, Kishinev, Republic of Moldova \\ ${ }^{2}$ International Laboratory of High Magnetic Fields and Low Temperatures, 53-421 Wroclaw, Poland \\ E-mail: condrea@lises.asm.md
}

Received October 12, 2009

\begin{abstract}
We studied the influence of uniaxial deformation on the transport properties of bismuth wires in the wide range of temperatures. Measurements of the resistance of bismuth nanowires with several diameters and different quality reveal oscillations on the dependence of resistance under uniaxial strain at $T=4.2 \mathrm{~K}$. Amplitude of oscillations is significant (38\%) at helium temperature and becomes smearing at $T=77 \mathrm{~K}$. Observed oscillations originate from quantum size effect. Evaluation of period of oscillations allows us to identify the groups of carriers involved in transport. Calculated periods of 42.2 and $25.9 \mathrm{~nm}$ satisfy approximatively the ratio 2:1 for two experimentally observed sets of oscillations from light and heavy electrons.
\end{abstract}

PACS: 73.63.Nm Quantum wires;

73.90.+f Electronic structure and electrical properties of surfaces, interfaces, thin films, and lowdimensional structures.

Keywords: bismuth; electrical resistance; quantum size oscillations; nanowires.

\section{Introduction}

A lot of the investigations concerned with various nanostructures is motivated partially by the very interesting thermoelectric and magnetotransport properties of bismuth nanowires (NWs) that make them potentially useful for device applications. Theoretical calculations [1-3] predicted that Bi nanowires should have an enlarged thermoelectric figure of merit, which results from the quantum size effect, have induced extensive studies of Bi NWs. Under the quantum size effect (QSE), several fundamental macroscopic characteristic of solids exhibit an anomalous dependence on reduced size. Therefore, for subsequent applications, a precise determination of the size-dependent parameters of investigated nanostructures is required. If the decreased size of wires or films becomes comparable with the electron wavelength $(d \sim \lambda)$, the transverse motion of carriers is quantized. Thus, the energy spectrum splits into subbands. When the discreteness of the energy subbands becomes significant, an oscillatory behavior of electron and hole density of states (DOS) as a function of thickness is expected for metal films $[3,4]$. Oscillations of DOS are due to variation in number of the subbands with diameter. According to the theoretical considerations of the QSE [35] all the transport coefficients which depend on the DOS should oscillate as a function of sample thickness with the period:

$$
\Delta d=h / 2\left(2 m_{t}^{*} E_{F}\right)^{1 / 2},
$$

where $m_{t}^{*}$ is the transverse effective masses, and $E_{F}$ is Fermi level.

The main experimental results in the investigations of the QSE have been obtained for thin semimetal films. The first quantum size oscillations in the resistivity, Hall coefficient, and magnetoresistance with a period of $400 \AA$ were observed in thin bismuth films [6]. Investigations [7] of bismuth films in large range of thickness (200-3000 $⿱$ ) revealed that the period of the resistance oscillations varied from 40 to $250 \AA$ with sample thickness. The difference in the period was attributed to the differences in the carrier concentrations due to growth conditions.

Quantum oscillations in the resistance of bismuthantimony alloy films were registered under variation in both thickness and $\mathrm{Sb}$ concentration at a fixed thickness [8]. The concentration oscillations in a sample with constant thickness are explained by the change of the transverse quasi-momentum caused by composition variation in the Bi-Sb alloy. An other manifestation of the QSE [9] was observed while studying the thickness dependence of the 
ratio of the electron and hole density of states measured under electric field effect (EFE). The method allows a rather precise determination of the film thickness period of the oscillations, which is about $370 \AA$.

In most of the above-mentioned cases, QSE was shown as an oscillatory behavior of the resistance dependence on film thickness. As is pointed out in [5], a variation in the value of the band overlap should also produce an oscillatory behavior of the kinetic coefficients. The changes in band overlap in a bulk Bi samples under deformation was described by Brandt [10]. The influence of the deformation on the band overlap changes was tested for bismuth films condensed on mica substrates [11]. Observed non-monotonous behavior of the resistance in bismuth films under sagging deformation is in a good agreement with the concept of QSE.

To our knowledge, up to now, most of the studies on quantum oscillations in transport properties of $\mathrm{Bi}$ nanostructures are concerned with thin films. The conditions of observation of the QSE on thickness dependences of the kinetic coefficients of thin wires are complicated by difficulties in the preparation of a series of samples with a small increment in thickness and identical characteristics of the bulk. Despite a lot of recently developed techniques for preparation of nanowires $[1,2,12]$ the bulk characteristic data from different experiments depend not only on sample cross-sectional dimensions and crystallographic orientation, but also on sample quality and purity, shell/matrix material and annealing treatment. It is possible to observe the oscillations of kinetic parameters, due to size-quantized energy spectrum on the individual cylindrical nanowire under certain external influence, for example, by impurity doping or deformation.

The aim of this study was to study transport coefficients of individual bismuth nanowires under condition of QSE by applying uniaxial stress for tuning the electronic structure and inducing band overlap changes. According to the condition of QSE realization $(d \sim \lambda)$, an oscillating behavior in transport coefficient of Bi samples is expected at diameters below $100 \mathrm{~nm}$. One should note that uniaxial strain in Bi NWs promotes the increases of band overlap between L-electron pockets and T-holes pocket at constant band gap, in contrast to its decrease under QSE.

\section{Experimental methods and samples}

Long Pyrex-coated Bi wires were fabricated using the same improved variant of the Taylor method. This method, which is presently known as the glass-coated melt spinning method, consists in the melting of a metal in a glass tube by rf induction heating and drawing a glass capillary in which the molten metal is entrapped.

The wire axis is at an angle of about $19^{\circ}$ with the bisector axis $C_{1}$ in the bisector-trigonal plane $C_{1} C_{3}$. This orientation is the same as that observed in $\mathrm{Bi}$ nanowire arrays by
Z. Zhang et al. [1]. Due to high elasticity of Pyrex capillaries, the limit of elastic stretching of the glass-coated $\mathrm{Bi}$ wires attains $\varepsilon=3.5 \%$ (in comparison for Bi whiskers $\varepsilon=$ $=2.0 \%$ [13] and for bulk Bi samples $\varepsilon=0.4 \%$ [14]). Due to small dimensions of glass-coated NWs, it was not possible to apply mechanical loading directly to them. For the measurements under uniaxial strain, the glass-coated wires with $d=70-150 \mathrm{~nm}$ and the length $L_{0}=2.0-3.0 \mathrm{~mm}$ were mounted on an elastic bronze ring in a special insert with stretching device similar to the method, used for whiskers [13]. The stretching was directed along the wire axis, i.e., close to the bisector axis $C_{1}$. The measurements of resistance were performed using two-probe method. Resistance variation was noted as $\Delta R / R=\left(R-R_{0}\right) / R_{0}$ where $R_{0}$ is the value of resistance in non-deformed state. Strain variation was noted as $\varepsilon=\left(L-L_{0}\right) / L_{0}$ where $L_{0}$ is the length of the wire in non-deformed state. Electrical contacts to the wire ends were made by Wood's alloy. Low dc currents $(0.1 \mu \mathrm{A} \leq I \leq 1 \mu \mathrm{A})$ were used to make sure that the voltage of the sample was a linear function of the applied current.

\section{Results and discussion}

The studies of the strain dependences of the resistance $R(\varepsilon)$ at $4.2 \mathrm{~K}$ for wires with various diameters revealed the oscillating dependence of the resistance on applied deformation in wires with a diameter of $90 \mathrm{~nm}$ subjected to thermal annealing (Fig. 1). Since an oscillating behavior in transport coefficient of $\mathrm{Bi}$ wires is expected at diameters below $100 \mathrm{~nm}$, we can suppose that observed oscillations are due to QSE. To our surprise, non-discernable and nonreproducible quantum oscillations on $R(\varepsilon)$ were observed in the thinner NWs with a diameter of $70 \mathrm{~nm}$. The dependences of resistance versus uniaxial strain $R(\varepsilon)$ for $\mathrm{Bi}$ wires with the diameters $d \geq 100 \mathrm{~nm}$ do not exhibits any oscillations and is similar to the one observed by us for

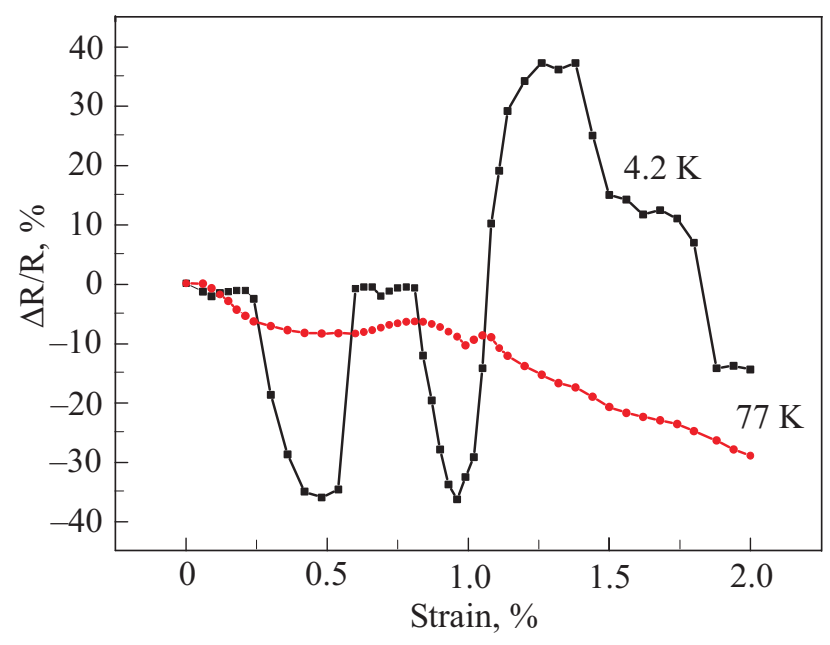

Fig. 1. Relative variation of the resistance as a function of applied strain for $90 \mathrm{~nm}$ Bi nanowire measured at $4.2 \mathrm{~K}$ and $77 \mathrm{~K}$. 


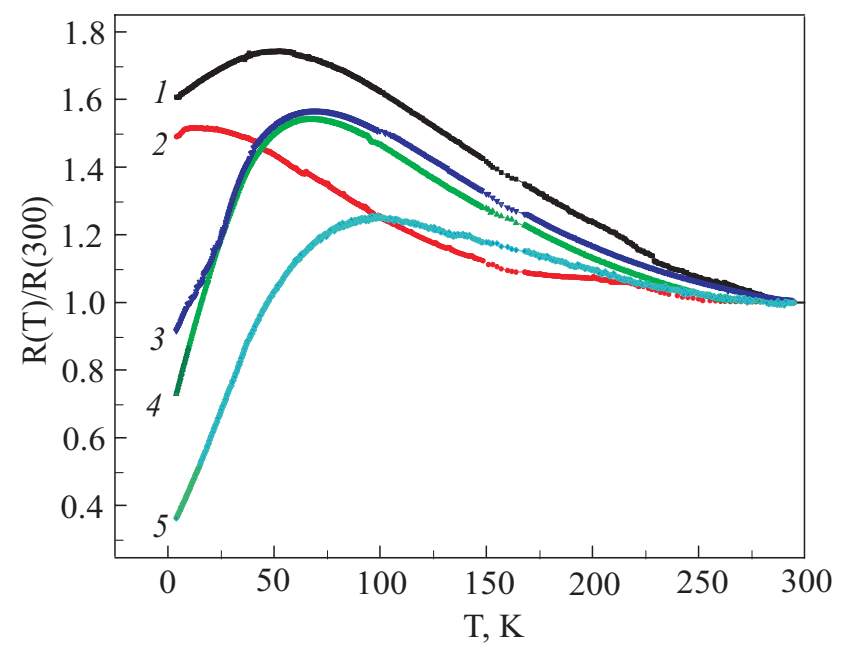

Fig. 2. Temperature dependence of resistance normalized to the resistance at $300 \mathrm{~K}$ for as-prepared Bi NWs: $d=90 \mathrm{~nm}(1), d=$ $=120 \mathrm{~nm}(3)$ and thermal annealed: $d=90 \mathrm{~nm}(2), d=120 \mathrm{~nm}$ (4), $d=150 \mathrm{~nm}$ (5).

thicker wires [15] and whiskers [13].

Figure 2 shows the temperature dependences of the electrical resistance $R(T)$ for Bi NWs with various diameters. Observed $R(T)$ dependences are consistent with previous results for $\mathrm{Bi}$ wires [12] and $\mathrm{Bi}$ nanowire arrays [1]. Curve 2 in Fig. 2 presents $R(T)$ dependence for a $90 \mathrm{~nm}$ NW after thermal treatment. History of the thermal treatment was not simple. Various variants of thermal treatment were tested and the optimal one was chosen. The NWs were annealed at $180^{\circ} \mathrm{C}$ for $10 \mathrm{~h}$ under vacuum with slow cooling back to room temperature. An evident increase of value of a residual resistance ratio (RRR) for the $90 \mathrm{~nm}$ wire after thermal treatment testified to an improved quality of annealed NWs. This supports the idea that a semiconducting behavior of $R(T)$ does not imply that a band gap opens even in the thin wires, a negative TCR may be rather an evidence of large defect density inside the as-prepared wires.

If we attribute the presence of oscillations on $R(\varepsilon)$ in Fig. 1 to the manifestation of QSE, in addition to the main condition $(d \sim \lambda)$ for QSE occurrence [4], we should consider some important requirements such as a small washing out of the discrete energy spectrum:

$$
h / \tau<<\Delta E,
$$

and a small temperature smearing:

$$
k T<<\Delta E,
$$

where $\tau$ is the relaxation time, taking into account scattering both in volume and on the surface; $\Delta E$ is the distance between discrete energy subbands. Inequality (2) means a high mobility and/or large free path length for carriers - involved in transport. It seems that all the requirements for QSE are satisfied for annealed $90 \mathrm{~nm}$ wire at $T=4.2 \mathrm{~K}$.

The observed resistance oscillations were reproduced on all the wires with a diameter of $90 \mathrm{~nm}$ from the series of samples subjected to thermal annealing. As one can see from Fig. 1, the $R(\varepsilon)$ dependence at $4.2 \mathrm{~K}$ exhibits a modulating profile consisting of two sets of oscillations with different periods determined by different groups of carriers. First, we supposed that two different periods of oscillations are due to contribution of electrons and holes to electrical conductivity. It is known that difference in the periods of electron and hole quantum oscillations results from the anisotropic properties of $\mathrm{Bi}$.

A rough evaluation of possible periods of quantum oscillations in $90 \mathrm{~nm}$ bismuth wire was made by formula (1). The value of effective transverse mass of heavy and light electrons was used from the model of the subband structure calculated in [1] for the Bi nanowire arrays with the same crystal orientation, corresponding to [1011]. This is our case where three groups of carriers operate: heavy electrons from two equiva-
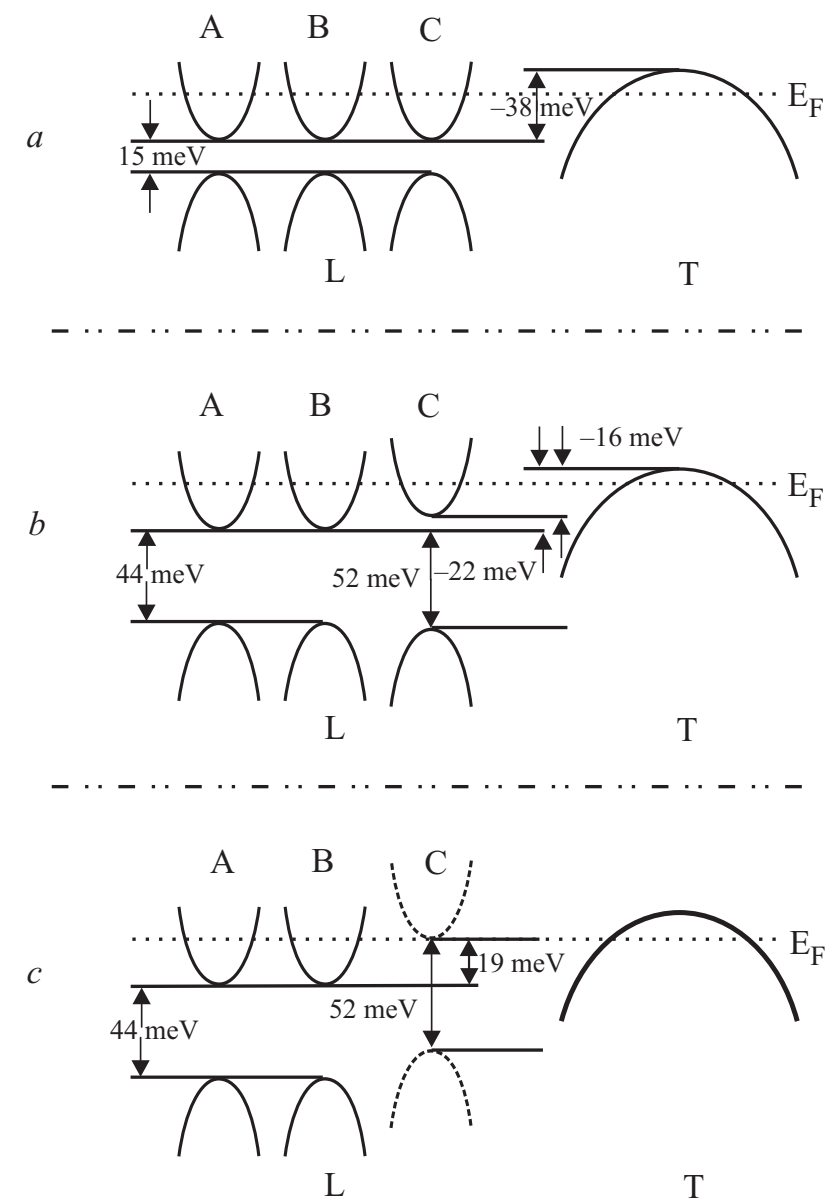

Fig. 3. Schematic diagram of $\mathrm{Bi}$ band structure at $L$-point and $T$-point near Fermi energy level: for bulk Bi (a); for $90 \mathrm{~nm} \mathrm{Bi}$ NW calculated in model [1] in non-deformed state $(b)$; a possible band structure for $90 \mathrm{~nm} \mathrm{NW}$ at the strain $\varepsilon \geq 0.96 \%$ (c). 
lent pockets $A$ and $B$, light electrons from pockets $C$, and heavy $T$-holes. According to [1], as the diameter of Bi nanowire arrays decreases below $100 \mathrm{~nm}$, the band overlap between light and heavy electron pockets and $T$-hole pocket decreases in the different ways, thus resulting in the splitting of the $L$-point band edge as shown in Fig. 3,b. In this model, the band edge energy of each subband is determined by the average transverse effective mass, approximated by the appropriate effective cyclotron mass. The values of effective masses calculated by authors of [1] are: $m^{*}=0.00212 m_{0}$ and $m^{*}=0.00372 m_{0}$ for light and heavy electrons, respectively. Estimated periods for our $90 \mathrm{~nm} \mathrm{NW}$ are: $\Delta d_{1}=42.2 \mathrm{~nm}$; $\Delta d_{2}=25.9 \mathrm{~nm}$ and $\Delta d_{3}=16.0 \mathrm{~nm}$ for light and heavy electrons and holes, respectively.

Coming back to measured quantum oscillations of resistance (Fig. 1) we note the period ratio of 2:1 for two set of observed oscillations. Two of calculated periods $42.2 \mathrm{~nm}$ and $25.9 \mathrm{~nm}$ satisfy approximatively the ratio $2: 1$. The calculated value of $16.6 \mathrm{~nm}$ for hole period is overestimated as a result of using the value for hole transverse mass $m^{*}=0.21 m_{0}$ available for bulk Bi. The absence of quantum oscillations from holes can be caused by its small period and non-detectable amplitude versus background. Thus, we can suppose that quantum oscillations with large amplitude and period of $42.2 \mathrm{~nm}$ occur from the light electrons with smallest effective mass, and quantum oscillations with weak amplitude and period of $25.9 \mathrm{~nm}$ occur from heavy electrons from pockets $A$ and $B$. A significant amplitude $(38 \%)$ of resistance quantum oscillations with $\Delta d_{1}=42.2 \mathrm{~nm}$ is due to the low quantum numbers of the subbands located below Fermi energy level in the pocket $C$ with light electrons. The vanishing of these oscillations at the deformation higher than $1 \%$ is suggestive of a change in the Fermi surface topology, known as an electronic topological transition (ETT) or Lifshitz transition. As a result of our previous investigations [15] of the changes in the Fermi surface topology under stress by means of Shubnikov-de Haas oscillation measurements, we have found the upward shift of the light electron pocket $C$ relative to the other two pockets $A$ and $B$ up to its complete vanish at a high strain. The shifted electron pocket is schematically indicated by the dashed line in Fig. 3,c. Due to the continuing increase in the band overlap between $L$-electron pockets $(A$ and $B)$ and $T$-hole pocket, quantum oscillations with a period of $25.9 \mathrm{~nm}$ are detected at a strain higher than $1 \%$.

If we attribute the presence of the oscillations on $R(\varepsilon)$ to the QSE manifestation, it is natural to make a brief analysis of correlation between the value of uniaxial strain and the period of observed oscillations $\Delta d$ in $90 \mathrm{~nm}$ wires. Though the precise interrelation between applied uniaxial strain and $\Delta d$ is not known, we try to follow the influence of strain on the Fermi level shifting through quantized subbands in electron pockets. In terms of previous results on ETT with the vanishing of one electron pocket, the work of extension of $\varepsilon=0.96 \%$ should be on the order of the energy shift of electron pocket $\mathrm{C}$ with strain. Since the measurements were made in the elastic deformation range, we may use the value of the elastic modulus for bulk Bi crystal along the bisector direction; thus, it is possible to determine the tensile load dependence $P$ on the value of extension $\varepsilon=\Delta L / L_{0}$ which is approximatively $P=0.38 \mathrm{GPa}$ at $\varepsilon=0.96 \%$.

A rough estimation of value of the energy shift was made by using the values of shifting rate $d E / d F$ of electron pockets under anisotropic deformation along the bisector direction for bulk Bi crystals calculated by authors in [17], which are: $d E / d F=0.5 \mathrm{meV} / \mathrm{kg}$ for pocket $C$ and $d E / d F=-0.6 \mathrm{meV} / \mathrm{kg}$ for pockets $A$ and $B$. In our case, the value of energy shift was determined at a strain of $0.96 \%$, where quantum oscillations with large period disappear. Calculated value is $d E \approx 19 \mathrm{meV}$. Because of some involved calculation uncertainties, the value of $d E \approx$ $\approx 19 \mathrm{meV}$ is rather appreciative; nevertheless, it is reasonable of the same order with value of Fermi energy level and of the band overlap (16 meV) for light electron pocket from the model of electronic band structure for $90 \mathrm{~nm} \mathrm{Bi}$ NWs, advanced by authors of [1].

\section{Conclusions}

Systematic measurements of the resistance of bismuth nanowires with several diameters and different quality reveal oscillations on the dependence of resistance under uniaxial strain at $T=4.2 \mathrm{~K}$. Amplitude of oscillations is significant $(38 \%)$ at helium temperature and becomes smearing at $T=77 \mathrm{~K}$. Observed oscillations originate from quantum size effect.

The absence of quantum size oscillations in resistance dependence for $70 \mathrm{~nm}$ wires can be explained partially by scarce number of light electrons responsible for oscillations with decreasing diameter [1] and partially by imperfection of the nanowires.

A simple evaluation of period of oscillations allows us to identify the groups of carriers involved in transport. Calculated periods of 42.2 and $25.9 \mathrm{~nm}$ satisfy approximatively the ratio 2:1 for two experimentally observed sets of oscillations from light and heavy electrons.

The importance of the quantum size effect manifestation in the resistance dependence on strain goes beyond studying the structure of electron spectrum, it can also be applied to investigate the spectrum of phonons. With a view to elucidate some aspects of the practical use of nanowires, we plan the further investigations under strain of the thermopower, which at low temperature may be due to the diffusive or phonon drag mode of carrier interaction.

1. Z. Zhang, X. Sun, M.S. Dresselhaus, J.Y. Ying, and J. Heremans Phys. Rev. B61, 4850 (2000).

2. Y.-M. Lin, X. Sun, and M.S. Dresselhaus, Prys. Rev. B62 4680 (2000). 
3. V.B. Sandomirskii, Zh. Eksp. Teor. Fiz. 52, 158 (1967). [Sov. Phys. JETP 25, 101(1967)].

4. B.A. Tavger and V.Ya. Demikhovskii, Usp. Fiz. Nauk 96, 61 (1968) [Sov. Phys. Usp. 11, 644 (1969)].

5. E.F. Schulte, Surf. Sci. 55427 (1976).

6. Yu.F. Ogrin, V.N. Lutskii, and M.I. Elinson, Pis'ma Zh. Eksp. Teor. Fiz. 3, 114 (1966) [Sov. Phys. JETP Lett. 3, 71 (1966)].

7. E.P. Fesenko, Fiz. Tverd. Tela 11, 2647 (1969) [Sov. Phys. Solid State 11, 2135 (1970)].

8. E.I. Bukhshtab, Yu.V. Nikitin, and Yu.F. Komnik Fiz. Nizk. Temp. 3, 755 (1977) [Sov. J. Low Temp. Phys. 3, 366 (1977)].

9. A.V. Butenko, Dm. Shvarts, V.B. Sandomirskii, and Y. Schlesinger, Physika B284-288, 1942 (2000).
10. N.B. Brandt, E.S. Itskevich, and N.Ya. Minina, Zh. Eksp. Teor. Fiz. 47, 455 (1954).

11. V. N. Lutskii, Phys. Status Solidi A1, 199 (1970).

12. T.W. Cornelius, M.E. Toinil Molares, R. Neumann, and S. Karin, J. Appl. Phys. 100, 114307 (2006).

13. Yu.P. Gaidukov, N.P. Danilova, and M.V. ShcherbinaSamoilova, Zh. Eksp. Teor. Fiz. 77, 2125 (1979) [Sov. Phys. JETP 50, 1018 (1979)].

14. O.P. Hansen, I.F. Mikhail, M.Yu. Lavrenyuk, and N.Ya. Minina, J. Low Temp. Phys. 95, 481 (1994).

15. P.P. Bodiul, V.F. Garabazhin, E.P. Condrea, and A.A. Nikolaeva, Czech. J. Phys. 46, 2417 (1996).

16. M. Gurvitch, J. Low Temp. Phys. 38, 777 (1980).

17. E.V. Bogdanov, M.Yu. Lavrenyuk, and N.Ya. Minina, Fiz. Tehn. Pol. 22, 1348 (1988). 